\title{
Conservative management of peri-apical cementoblastoma: A case report
}

\author{
Fahad Quadri $^{1 *}$, Saara Khiyani ${ }^{2}$, Sana Quadri $^{3}$, Pallavi Bhailume ${ }^{4}$, Vidya Patil $^{5}$ \\ ${ }^{1}$ Lecturer, ${ }^{2}$ Reader, ${ }^{3}$ Clinical Assistant, ${ }^{4}$ Assistant Professor, ${ }^{5}$ Post Graduate Student, Dept. of Conservative Dentistry \& \\ Endodontics, ${ }^{1,2} \mathrm{CSMSS}$ Dental College, Aurangabad, ${ }^{3-5} \mathrm{GDC} \& \mathrm{H}$, Aurangabad, Maharashtra, India
}

\section{*Corresponding Author: Fahad Quadri}

Email: dr.fquadri@gmail.com

\begin{abstract}
The case report describes the diagnosis and endodontic treatment of tooth followed by surgical management by apicoectomy as an effective measure for the treatment of cementoblastoma. This case report presents treatment other than extraction of tooth diagnosed with cementoblastoma A 33 year's old female patient presented dull aching pain in the lower right back region of the jaw. On clinical mild attrition and tenderness were seen with 45 on palpation bony expansion was observed in the vestibule area of $45 \& 46$. The pulp vitality test gave no response with 45 and delayed responses with 46 . Radiographically dense cemental thickening was seen with 45 and hyper-sclerosis of bone in the periapical region on 46 . CBCT (cone beam computer tomography) evaluation revealed buccal bone expansion and buccal cortical bone resorption with 45. Apicoectomy was performed after 6-months of endodontic management of 45. The histological evaluation confirmed the diagnosis of cementoblastoma. Clinical radiograph after $6 \& 10$ months suggested periapical healing with the absence of signs and symptoms. However, the patient must be kept on follow up for the possibility of recurrence of this lesion. The importance of these findings demonstrated that the treatment of cementoblastoma may be conservative with the maintenance of the affected teeth.
\end{abstract}

Keywords: Dental cementum.

\section{Introduction}

Dental cementum is a specialized connective tissue that generally covers the entire root surface from the Cemento-Enamel Junction (CEJ) to the root apex. The cementoblastoma is a benign odontogenic tumor of ectomesenchymal origin that preferentially affects the roots of mandibular molars or premolars and has a slight prevalence in the male sex population. ${ }^{1}$ Clinically it is characterized by slow-growing bone expansion, with no cortical bone destruction. The tumor may or may not be associated with pain. ${ }^{2}$

Cementoblastoma can resemble radiographically with hypercementosis. It is the thickening or increase in the deposition of cementum on the root surface that may involve the entire root or appear nodular or bulbous around the root apex..$^{3-4}$ The etiology and treatment options are somewhat limited, with reports regarding hypercementosis and its endodontic management as being sparse. ${ }^{5}$

\section{Case Report}

A 32 years old female came to dental college \& hospital with the chief complain of dull aching pain in the lower right back region of jaw since one month. The pain was intermittent and aggravated on mastication. Analgesics provided temporary relief to the patient. She didn't have any history of systemic disease and was not on any medication. On examination, mild attrition was seen with 45 (FDI notation). Slight tenderness was evident with $45 \& 46$ without mobility. While palpating the muco-buccal fold a mild bone expansion was felt adjacent to $45 \&$ 46. The pulp vitality test suggested no response with 45 and delayed response with 46.

IOPA (intra-oral peri-apical) radiographs suggested radiopaque mass around the apical third of 45(Fig. 1-A) and radiopaque sclerosis of bone in the periapical region on 46(Fig. 1-A). The CBCT images suggested that the radiopaque mass is continuous with apical dentin of 45 . The scans also suggested radiolucent lesions and loss of lamina dura with 45 and 46 (Fig. 1-H). CBCT evaluation expressed buccal 
cortical bone expansion \& its erosion with 45(Fig. 1$\mathrm{G})$. Minute communicating lines were seen with periapical lesions of $45 \& 46$ (Fig. 1-H). Hence it was decided to undergo Root Canal Treatment with $45 \&$ 46. During treatment, the working length couldn't be determined by electronic apex locator with 45 . Calcium hydroxide intracanal medicament placed for 10days along with the routine disinfection procedure was advocated. The root canal treatment was completed in 3 visits \& the patient was put on followup visits every month.

The symptoms subsided as soon as the procedure was over \& the periapical surgery was planned. The patient was not willing to opt for surgery due to absence on symptoms but the previous symptoms again reappeared after 6 months. CBCT and IOPA suggested aggravation of the periapical lesion with 35 .

Apicoectomy and curettage was planned with 45 (Fig. 2-A). Some part of the root was removed along with the cemental mass and was sent for histopathological analysis (figure2-B). The histopathological evaluation suggested irregular scalloped borders with clastic activity at the periphery $\&$ numerous lacunas and reversal lines are seen with hard tissues. All this histopathological finding suggested Cementoblastoma. Post-operative evaluation after 6 \& 10-month IOPA suggested resolving periapical lesions (Fig. 1-E, F). Postoperative lip numbness did appear but was resolved within 1 month.

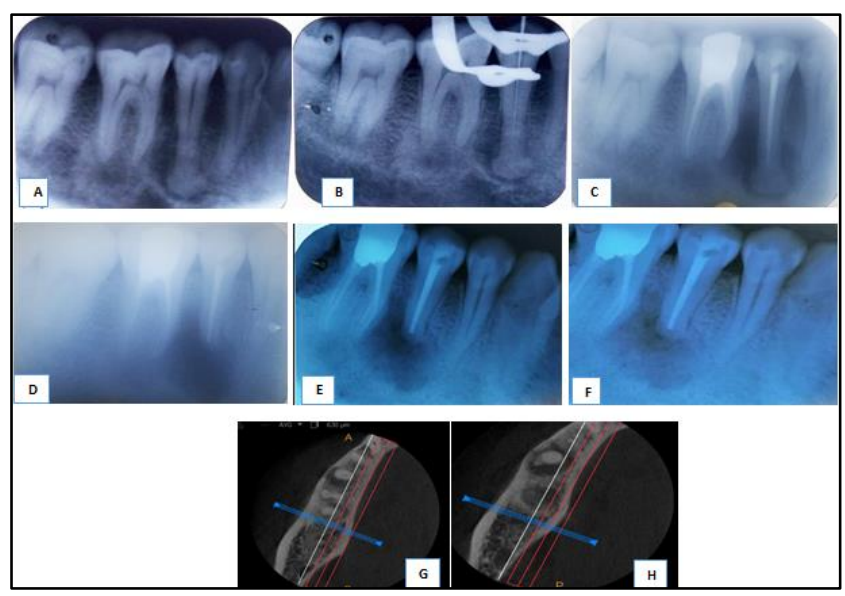

Fig. 1: Diagnosis and endodontic treatment. A: Preoperative IOPA 45. B: IOPA showing working length determination. C: Post endodontic treatment IOPA of
45 \& 46. D: Post surgical IOPA with 45. E: 6 months follow up IOPA with 45. F: 10 months follow up IOPA with 45. G: Axial view of CBCT buccal bone expansion with resorption. H: Axial view of CBCT showing faint communication seen between periapical lesions of $45 \& 46$.

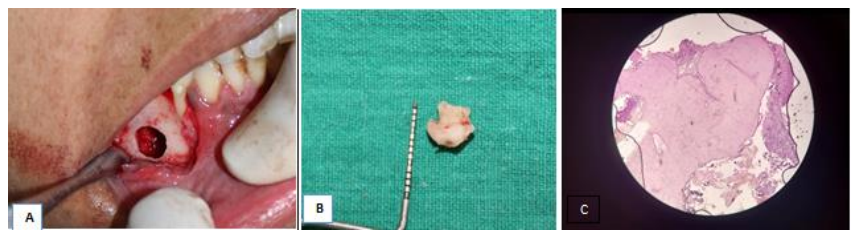

Fig. 2: Surgical and histological images. A: surgical access for apicoectomy with 45 . B: cemental mass along with root protion. C: Histological evaluation displaying prominent basophilic reversal lines and cementoblastic rimming \& numerous scattered multinucleated giant cells are seen.

\section{Discussion}

Cementum is an adaptable component of the periodontium, which can respond to functional changes during its development, with its formation being characterized by apposition during the final stage of the tooth's life. Radiographically, hypercementosis consists of a well-defined dense radiopaque mass fused with one or more roots and surrounded by a thin radiolucent line and no clinical signs and symptoms are seen. ${ }^{7}$

Despite the name benign the aggressive form of cemental defect known as cementoblastoma, it is characterized by signs such as root resorption, loss of root contour, disruption of lamina dura, expansion/erosion of cortical bone, pain and pulp vitality of the teeth is affected. ${ }^{7}$ Radiographically, this odontogenic tumor consists of a well-defined dense radiopaque mass fused with one or more roots and surrounded by a thin radiolucent line, thus simulating a hypercementosis. ${ }^{7}$ This data have helped us in the diagnosis of localized benign cementoblastoma.

The extension of the lesion in the periapical area of 46 which might have led to reduced vitality and symptoms with 46 , hence endodontic treatment was initiated with 45 \& 46. Due to morphological characteristics, it becomes difficult to determine \& establish the relationship between the external anatomy of the apical third region \& pose difficulty in 
measuring the working length during treatment procedures.

Surgical removal of cemental mass along with the removal of some part of root and apicoectomy is indicated after root canal treatment in cases of cementoblastoma due to its high rate of recurrence. ${ }^{1}$

Tooth extraction has also been suggested as a treatment modality for cementoblastoma due to its high recurrence rate associated with its incomplete removal. ${ }^{1}$ The majority of the studies in literature and this case report did not report relapses after 1-year recall, however, according to Brannon et al., after a 66 months follow-up, a 37\% recurrence rate was reported, thus emphasizing the need for a long-term monetarization. ${ }^{7}$ Chrcanovic BR. Et al. suggested that the recurrence rate of cementoblastoma was $11.8 \%$ \& the preservation of the involved teeth and the location of the lesion seem to not influence the recurrence rate. ${ }^{8}$

CBCT imaging provided a very useful aid in diagnosis and treatment. It not only aided in the diagnosis by revealing buccal cortical bone resorption which suggested cementoblastoma but also in planning by aiding in deciding the surgical extent as the apices of 45 is proximity to the mental foramen.

Post-operative lip numbness might be because of intra-tissue edema as during the surgery utmost care was taken to avoid trauma to the mental nerve. The lip numbness completely recovered in a month

\section{Conclusion}

The evaluation and correct diagnosis is the key to the success of the treatment. Conservative management of cementoblastoma is possible and should be opted before extraction. It's very clear that hypercementosis and cementoblastoma are distinct entities with similar clinical and imaging characteristics, which require completely different treatment measures, a more conservative for hypercementosis and a more extensive for cementoblastoma.

\section{Source of Funding}

None.

\section{Conflict of Interest}

None.

\section{References}

1. Costa BC, de Oliveira GJ, Chaves MD, da Costa RR, Gabrielli MF, Guerreiro-Tanomaru JM, Tanomaru-Filho M. Surgical treatment of cementoblastoma associated with apicoectomy and endodontic therapy: Case report. World $J$ Clin Cases 2016;4(9):290.

2. Goerig AC, Fay JT, King E. Endodontic treatment of a cementoblastoma: report of a case. Oral surg, Oral Med, Oral Pathol 1984;58(2):133-6.

3. Zhou J, Zhao Y, Xia C, Jiang L. Periodontitis with hypercementosis: report of a case and discussion of possible aetiologic factors. Aust Dent J 2012;57(4):511-4.

4. Bürklein S, Jansen S, Schäfer E. Occurrence of hypercementosis in a German population. J Endod 2012;38(12):1610-2.

5. Consolaro A, Consolaro RB, Francischone LB. Hypercementosis andincreased cementum thickness over the age: Clinical implications and meanings. Dent Press Implantol 2012;6(1):20-32.

6. Pinto AS, Carvalho MS, de Farias AL, da Silva Firmino B, da Silva Dias LP, Neto JM et al, Hypercementosis: Diagnostic imaging by radiograph, cone-beam computed tomography, and magnetic resonance imaging. J Oral Maxillofac Radiol 2017;5(3):90.

7. de Andrade MG, de Paula DM, Quintas PH, de Carvalho MM, Júnior BC, de Azevedo RA et al. Benign Cementoblastoma-A Rare. JSM 2018;6(2):1106.

8. Chrcanovic BR, Gomez RS. Cementoblastoma: An updated analysis of 258 cases reported in the literature. J CranioMaxillofac Surg 2017;45(10):1759-66.

How to cite this article: Quadri F, Khiyani S, Quadri $\mathrm{S}$, Bhailume P, Patil V. Conservative management of peri-apical cementoblastoma: A case report. $J$ Community Health Manag 2019;6(4):136-8. 